\title{
Stress Analysis and Study the Fracture Mechanics in Planetary Gear Set Using FEM
}

\author{
Hozhabr Mozafari ${ }^{1 *}$, Sarah Najafian ${ }^{2}$ \\ 1,2 Railway Engineering School, Iran University of Science and Technology, Narmak, Tehran, Iran, 16846-13114
}

\section{KEY WORDS}

Planetary gear set, LEFM, FEM, ABAQUS, Crack growth path, Contact stress, XFEM.

\begin{abstract}
Automotive and aerospace industries are relevant to use planetary gear sets headed for their incomparable features. Planetary gears flexibility in achieving different speed ratio and high-power-density design makes them often suitable to counter-shaft gear reduction systems. The main components are sun, ring and planet gears. Planet gears are engaged with sun and ring gears and subjected to the severe contact stresses. Consequently, gear tooth crack failure may occur when the critical values are reached. Hence assessment of strength due to contact stresses and failure phenomena is vital to avoid catastrophic damage to the system. Basic types of gear teeth damages include: surface deterioration, scuffing, permanent deformation, surface fatigue phenomena, cracks, and gear tooth fracture. Failure may occur by crack growth through the gear teeth or through the gear rim. These types of failures are caused excessive damage to the system and should be prevented. In this paper contact stress distribution between gears are investigated by mean of finite element method and compared to ISO standards. Then planet gear root crack failure analysis is carried out with the principles of linear elastic fracture mechanics. Fracture mechanics deals with the study of how a crack in a structure propagates under applied loads. For this purpose, Multistep analysis is used to determine stress intensity factors for different crack sizes to evaluate the crack propagation path, incrementally. Also, XFEM-LEFM based analysis performed to evaluate the efficiency of this method compared with contour integral method. Rim thickness effect has been studied to assess its influence on the crack growth path.
\end{abstract}

\section{Introduction}

Planetary gear sets are used in wide range of industries such as automotive and aerospace companies which are relevant to implement these types of gear sets in propulsion and power transmission systems. Because of flexibility in achieving different speed ratios and high power-density design and coaxial arrangement of the input and the output shafts, these kinds of gear systems play a very important role for power transmission. Therefore, due to special geometric properties and motion features, comprehensive information is needed to design and manufacturing. Accurate estimation of the strength of gears is essential to achieve appropriate performance, especially in contact positions, where gears teeth sustain high contribution of contact stresses. Although, possibility of crack growth increase in high stress domain, i.e., gear teeth, and cause failure in system. Hence, fracture phenomenon should be taken 
into account to assess the efficiency of the gear system. Micro cracks are commonly exist in structures because of nature of materials and when the structures are subjected to different loading conditions in their service life, macro cracks may appear and develop through the structure. Crack growth rate and propagation path are associated with the geometry and loading conditions. In a gear, crack mainly nucleated at root of gear tooth, which is subjected to intensive stresses. Gear tooth root crack will cause changes in vibration characteristics of gear system. Crack may propagate through a tooth or into the rim that causes catastrophic failure.

In this paper, stress analysis of a planetary set is carried out by using finite element method. For this purpose, two dimensional model of gear set has been created which contains sun, planet and ring gears to survey the strength of the gear set. According to ISO standards, calculations have been done and contact stresses obtained, respectively. FEM results were in good agreement with the calculated results, which validate the FE model. In the next step, planet gear root crack failure analysis is carried out with the principles of linear elastic fracture mechanics. Fracture mechanics deals with the study of how a crack in a structure propagates under applied loads. For this purpose, Multistep analysis is used to determine stress intensity factors for different crack sizes to evaluate the crack propagation path, incrementally.

\section{FEA Contact Stress Analysis}

Estimation of the gears strength and computation of contact stresses of involved teeth which are in contact are vital to assess the efficiency of the gear set. Several investigations were carried out to measure the contact forces of different types of gears. Wie [1], investigates the characteristics of an involute gear system including contact stresses, bending stresses, and the transmission errors of gears in mesh. He validates the FE model with the calculated results of Hertz's equations, which were originally derived for contact between two cylinders. Chen and Shao, [2], were working on tooth crack model and then extend it to include the effect of fillet-foundation deformation, and the effects of gear tooth crack on gear mesh stiffness in a spur gear. In this section, stress distribution in a planetary gear set is obtained by mean of finite element method. Two dimensional model of assembled gear set is created by ABAQUS software. In some previously researches about simulating the gear performance, it was common to simplify the model by only modeling the teeth in contact, [3]. For performing analogous simulation, alternative boundary conditions should be applied in boundary of teeth. This type of simulation is used to reduce the size of the problem and beneficial in most cases, such as spur gears. Although, in the planetary gear set, planet gears have both rotational and translational movements, and it is necessary to consider contact situations accurately; therefore it is not acceptable to use this approach. In this study, complete model of planetary gear set is developed in FE model, Figure. 1.

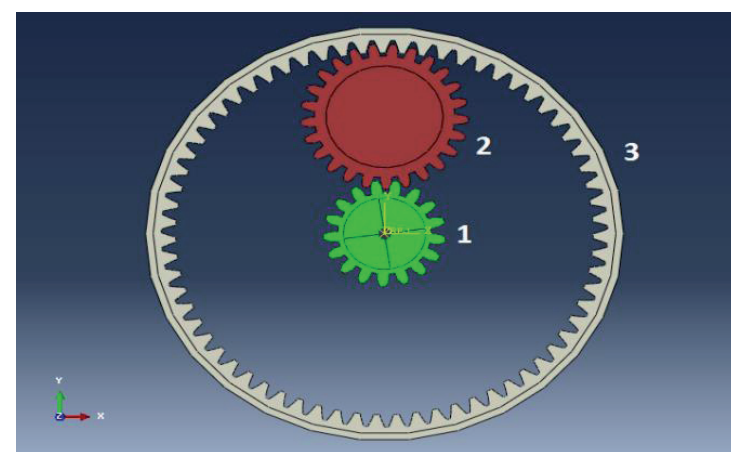

Fig. 1: Planetary gear set arrangement, 1-sun gear; 2-planet gear; 3-ring gear

In contact problems, the accuracy of obtained contact stresses is associated with the meshing of contact positions. Hence, gear teeth are meshed more refined as shown in Figure. 2.

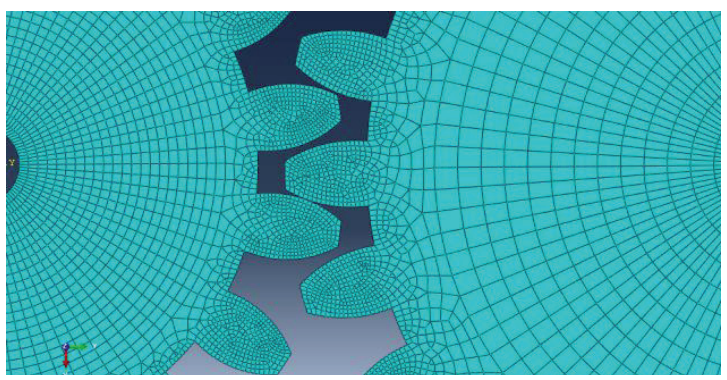

Fig. 2: Refined mesh on the gears teeth

Boundary conditions were applied to the ring and sun gears such that the ring gear was fixed and the sun gear allowed rotating around its center of rotation. Input torque applied to the sun gear. Abaqus/standard solver is used to calculate 
the contact stresses, by assumption of ignoring inertia effects in analysis. Standard solver is recommended for static and low-speed dynamic events where, highly accurate stress solutions are crucially needed. [4]

\section{Theoretical Analysis}

Analysis carried out by using ISO standards, [5]. Correspond to ISO 6336 contact stress equation defined as:

$S_{H}=z_{e} \frac{2}{d_{w}} \sqrt{\frac{2 T}{b_{w} \sin \left(2 a_{w}\right)} \frac{(u \pm 1)}{u}}$

Where

$\mathrm{z}_{\mathrm{e}}$ : Elasticity factor (for steel is $189.8 \mathrm{~N} \wedge 0.5 / \mathrm{mm}$ )

T: applied torque

$\mathrm{b}_{\mathrm{w}}$ : contact force width

u: Gear ratio

$\mathrm{d}_{\mathrm{w}}$ : pitch diameter

In a planetary gears, equation (1) is used defined the pitch point contact stress in the sun/planet gear mesh by

$S_{H 12}=z_{e} \frac{2}{d_{w 1}} \sqrt{\frac{2 T_{1}}{b_{w 12} \sin \left(2 a_{w 12}\right)} \frac{\left(u_{12} \pm 1\right)}{u_{12}}}$

And in sun/ring gear mesh

$S_{H 23}=z_{e} \frac{2}{d_{w 2}} \sqrt{\frac{2 T_{2}}{b_{w 23} \sin \left(2 a_{w 23}\right)} \frac{\left(u_{23} \pm 1\right)}{u_{23}}}$

When gear mesh losses neglected

$S_{H 23}=z_{e} \frac{2}{d_{w 2}} \sqrt{\frac{2 u_{12} T_{1}}{b_{w 23} \sin \left(2 a_{w 23}\right)} \frac{\left(u_{23} \pm 1\right)}{u_{23}}}$

Where the subscript indexes "12" and "23" are for the sun/planet gear and the planet/ring gear mesh, respectively .The specifications of the planetary gear set are shown in Table 1.

Table 1: Specifications of the planetary gear set.

\begin{tabular}{|l|l|l|}
\hline Gear & Teeth & Dia. $(\mathbf{m m})$ \\
\hline Sun & 16 & 64.5 \\
\hline Planet & 26 & 95.25 \\
\hline Gear & 68 & 269.2 \\
\hline
\end{tabular}

Applied torque 30000 (N.MM) is considered to the sun gear. The modulus of elasticity of gears is
207000 (Mpa) and Poisson ratio is 0.3. Figure 3, And Figure 4, shows the contact stresses of sun/planet and sun/ring respectively. It can be seen severe local stress exists in contact patches.

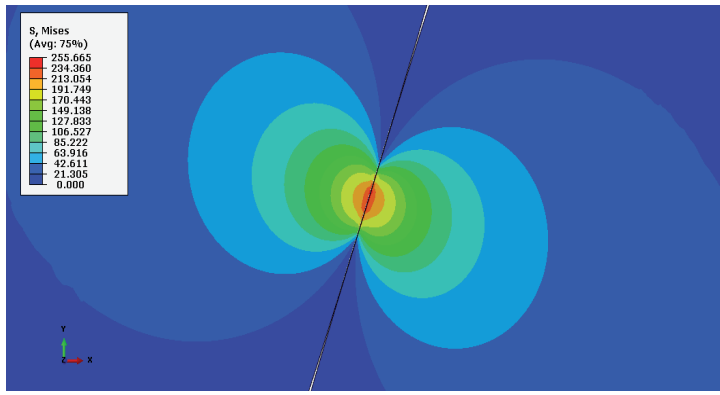

Fig. 3: Contact stress distribution of sun/planet gears.

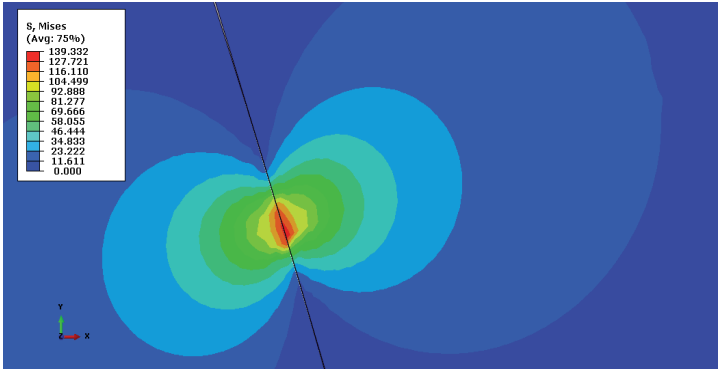

Fig. 4: Contact stress distribution of sun/ring gears.

Table 2, the results of analytical method validates the authors' FE model. These results obtained after several meshing attempts, which the mesh sensitivity curve is not presented here.

Table 2: Contact stresses comparison.

\begin{tabular}{|l|l|l|}
\hline & $\begin{array}{l}\text { Theoretical analy- } \\
\text { sis (MPa) }\end{array}$ & $\begin{array}{l}\text { FEM analysis } \\
\text { (MPa) }\end{array}$ \\
\hline SH12 & 255.47 & 255.66 \\
\hline SH23 & 136.39 & 139.33 \\
\hline
\end{tabular}

\section{LEFM-Contour Integral}

Fracture mechanics mainly used for predicting strength and life of cracked structures. Linear elastic fracture mechanics can be used to describe the behavior of cracks. The basic assumption of this theory is determining the crack growth behavior in elastic domain of material by using stress intensity factors. Therefore, calculating the SIFs is very important to achieve accurate results. In this step crack growth behavior on the planet tooth root have been studied. The accuracy and reliability of 
the analysis of a cracked body primarily depends upon the accurate determination and continuity of the crack path. It is therefore very important to select the crack growth criteria very carefully. Several criterions are used to obtain principal crack parameters such as; MTS criterion, SEDC criterion, $T$ criterion and $\mathrm{M}$ criterion. In this model maximum tangential stress criterion was used. Maximum tangential stress algorithm showed good results in predicting crack paths. Modeling stationary discontinuities, such as a crack, with the conventional finite element method requires that the mesh conforms to the geometric discontinuities. Therefore, considerable mesh refinement is needed in the neighborhood of the crack tip to capture the singular asymptotic fields adequately. Modeling a growing crack is even more cumbersome because the mesh must be updated continuously to match the geometry of the discontinuity as the crack progresses. Hence to speed up the analysis parametric model generated by using Python Script codes. To reduce the volume of problem Submodeling technique is applied to the model. The submodeling technique is capable of providing more accurate analysis of the stresses around the crack tip. The global model has a coarse mesh, while the submodel has a refined mesh, but the submodel boundary conditions must be same with the global model. The global model consists of sun, planet and ring gear. But planet gear imported to the submodel exclusively.

In MTS criterion, stress field near the crack tip for elastic and homogenous material is given by:

$$
\begin{aligned}
& \sigma_{\theta \theta}=\frac{1}{\sqrt{\pi \cdot r}} \cos \theta\left(K_{I} \cos \frac{1}{2} \theta^{2}-\frac{3}{2} K_{I I} \sin \theta\right)(5) \\
& \tau_{\mathrm{r} \theta}=\frac{1}{2 \sqrt{2 \pi \cdot r}} \cos \frac{1}{2} \theta\left[K_{I} \sin \theta+K_{I I}(3 \cos \theta-1)\right](6)
\end{aligned}
$$

Where $\mathrm{r}$ and $\theta$ are polar coordinates centered at the crack tip in a plane perpendicular to the crack front. $\mathrm{K}_{\mathrm{I}}$ Is open mode and $\mathrm{K}_{\mathrm{II}}$ is shear mode, respectively. The direction of crack propagation can be obtained by

$$
\phi=\cos ^{-1} \frac{3 K_{I I}^{2}+\sqrt{K_{I}^{4}+8 K_{I}^{2} K_{I I}^{2}}}{K_{I}^{2}+9 K_{I I}^{2}}
$$

Where $\phi$ is the crack growth angle.
According to the experiences about failure in gears, it is reported that most of the failures begins by a crack nucleation near the root of the gear tooth, Figure. 5.

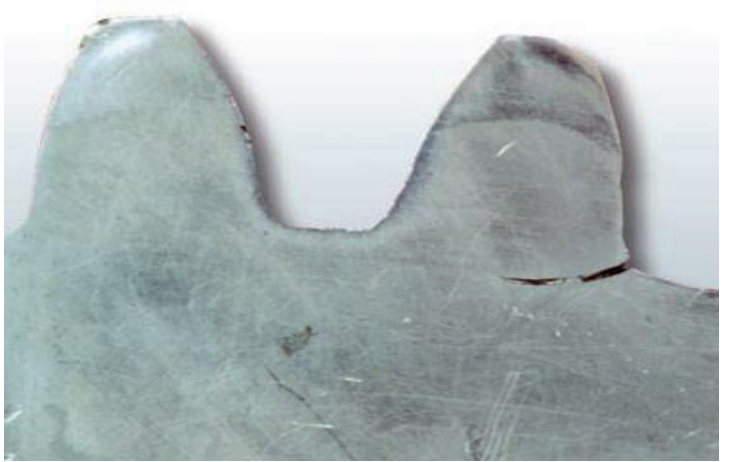

Fig. 5: Crack growth through the gear tooth

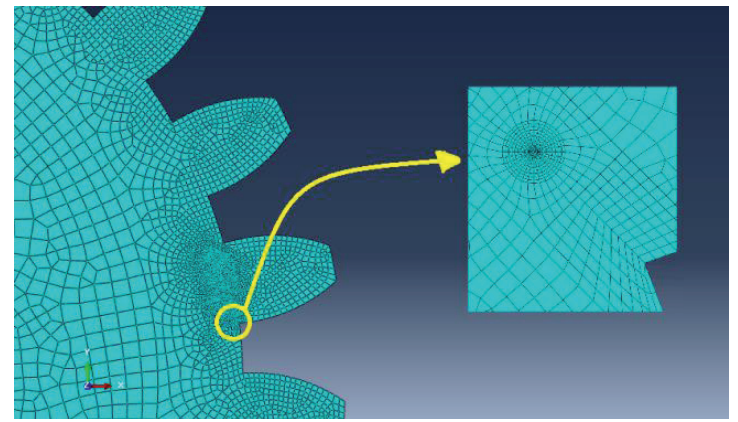

Fig. 6: Initial crack created on the root of planet gear tooth

These failures may results in noise and acoustic emissions that could be a warning for immediate handling or sometimes catastrophic failure which cause costly damage to the system, [6]. Planet gears play an important role in transmission of the applied load by sun gear to the carrier. These gears are subjected to both rotational and translational movements and exposed to severe contact stresses by both sun gear and ring gear. Therefore durability characteristics of planet gear should be considered to avoid noise and/or failure in the gear system. Hence, in this section fracture mechanics of planet gear has been studied by creating initial crack at the tooth root. In some numerical researches the gear held fixed through the analysis, but for planet gear applying such boundary condition may give unreliable results. Therefore, realistic contact should be used in this simulation. Refined mesh near crack region has been generated and singularity of crack tip is taken into account by mean of second order ele- 
ments, Figure. 6, virtual crack extension technique was used to calculate the stress intensity factors.

Crack growth modeled by performing multistep analysis, which in each step, new crack created and after remeshing, the growth path is predicted correspond to previous step results.

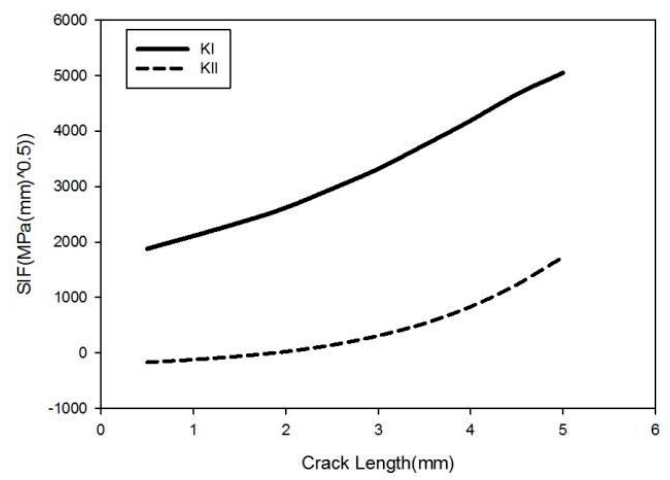

Fig. 7: Calculated stress intensity factors.

\section{XFEM-LEFM Based}

Contour integral method is costly compare to the approaches like Extended finite element method, due to the mesh generation in each step and correlation of crack location with the elements boundaries to make singularities. Extended finite element (XFEM) method offers an elegant way to model discontinuities and singularities independently of the mesh. XFEM is a partition of unity based method, where special functions describing the field behavior are incorporated locally into the finite element approximation space and the resulting space is fully capable of capturing all the features of interest. The XFEM methodology to locally enrich the field, where a local partition of unity is satisfied to capture the desired features of interest in the solution.

Modeling stationary discontinuities, such as crack, with the conventional finite element method requires that the mesh conforms to the geometric discontinuities. Therefore, considerable mesh refinement is needed in the neighborhood of the crack tip to capture the singular asymptotic fields adequately. Modeling a growing crack is even more cumbersome because the mesh must be updated continuously to match the geometry of the discontinuity as the crack progress. The extended finite element method alleviates the shortcomings associated with meshing crack surface. The extended finite element method is an extension of the conventional finite element based on the concept of partition of unity, which allows local enrichment functions to be easily incorporated into a finite element approximation. Because the mesh is not required to conform to the geometric discontinuities, the initial location of a pre-existing crack must be specified in the model. When the extended finite element method is used, the mesh is not required to match the cracked geometry. The presence of a crack is ensured by the special enriched functions in conjunction with additional degree of freedom. This approach also removes the requirement to explicitly define the crack front or to specify the virtual crack extension direction when evaluating the contour integral. Therefore another analysis was performed to compare the XFEM with contour integral results. In this analysis initial crack is also created to speed up the analysis. After several remeshing, it is preferred to refine mesh in a band through the gear tooth root where it is predicted for crack growth by previous results, Figure 8.

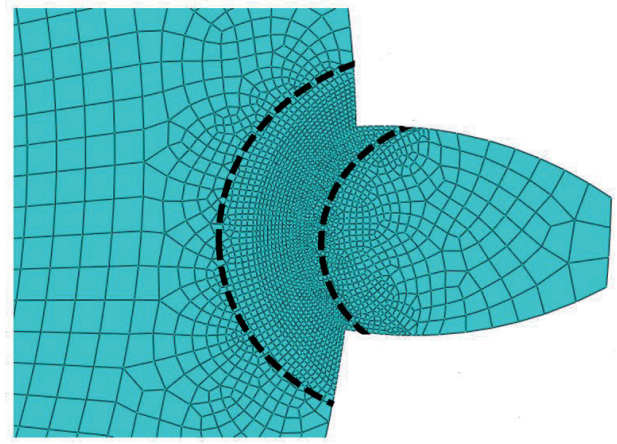

Fig. 8: Refined mesh through the gear root tooth.

As the XFEM method needs to develop the crack size by using enrichment point, overall fracture mechanics may become costly if the mesh generated not carefully, hence, by selecting a narrow band to refine mesh accurate results could achieved without severe CPU time.

\section{Effect of Rim Thickness}

A common design goal for gears in all of power transmissions such as helicopter, aircraft and etc., is to reduce weight. Typical method to reach this goal is rim thickness reduction. Therefore some gear design use thin rims. Rims that are too thin, however, may lead to bending fatigue problems. Depending on the geometry and load on the gear or the severity of the defect, a crack may propagate through a 
tooth or into the rim, this could lead to disengagement of a rotor or propeller from an engine, this type of failure should be avoided. On the other hand, a crack which propagates through a tooth may or may not be catastrophic. The severity of this failure mode would depend on design and operating conditions such as, contact ratio and whether the debris remained in mesh to jam the gear train. Also, ample warning of this failure mode may be possible due to advances in modern diagnostic systems. Proper tooth design can usually prevent gear tooth bending fatigue. However, gear tooth or rim failures may occur even when the tooth design itself is adequate. Possible causes of such failures are insufficient rim thickness in the design, improperly processed material containing inclusions where cracks can start, severe operation conditions such as overload or misalignment, operation near the resonant frequency of a gear structure, or localized wear such as fretting at a gear-shaft connecting joint which could initiate a crack. Then crack may propagate and cause failure.

To predict the type of failure, i.e., root and internal diameter failure, it is necessary to evaluate the effect of rim thickness on the crack growth path. Therefore, in this section analyses were performed respect to the different rim thicknesses to assess the effect of rim thickness on the crack growth path. In each step of analysis crack growth has been simulated to final fracture and similar method for other thicknesses was considered.

\section{Result and Discussion}

Contact stresses which are computed by FE model and analytical method are shown and compared in Table 2. The FE model results were in good agreement with the analytical results.

Figure. 7, shows the stress intensity factors variation for each increment of crack growth. It can be seen that $\mathrm{K}_{\mathrm{I}}$ is the dominant mode in crack growth. Therefore, opening mode has the most effective contribution in gear tooth failure, which is approximately neglecting any influence of shear. It is clear in Figure. 9, the predicted crack growth is along the root. Also, Figure. 10 illustrate that XFEM-LEFM based predicted crack growth path similar with LEFM-Contour integral method; however, in the first method it is not necessary to redefine mesh in each step of analysis and crack growth path calculated by enrichment points.

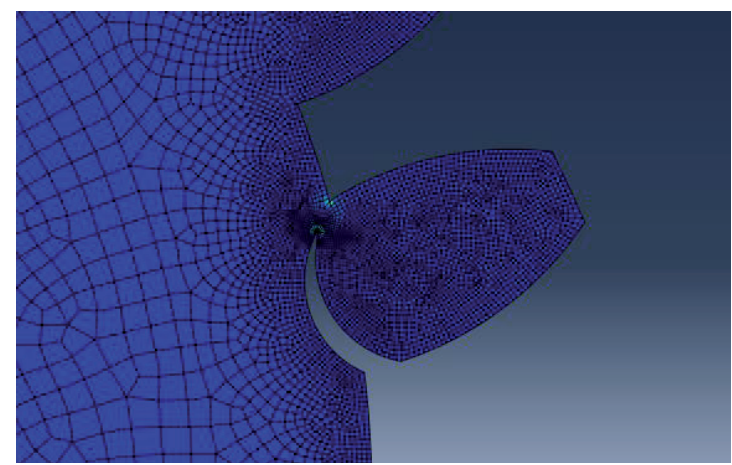

Fig. 9: Crack growth path predicted by MTS criterion.

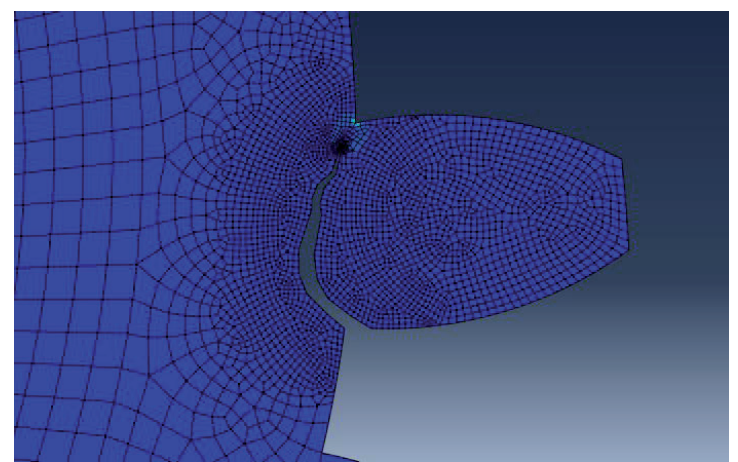

Fig. 10: Crack growth path predicted by XFEM-LEFM based method.

Also, the effect of rim thickness has been investigated by considering three main thicknesses. The results show that in thin rim the crack growth internally to the rim, which causes catastrophic failure, and should be prevented, Figure. 11.

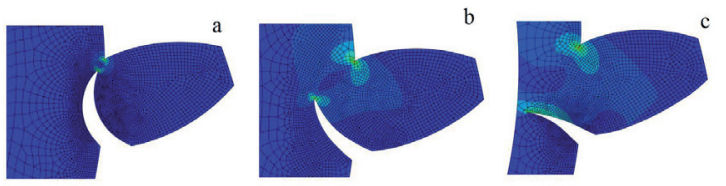

Fig. 11: Effect of rim thickness ( $T$ ) on crack growth path; a) $T=20 \mathrm{~mm}, b) T=10 \mathrm{~mm}, \mathrm{c}) \mathrm{T}=5 \mathrm{~mm}$.

\section{Conclusion}

The paper present a computational model for estimation the strength of a planetary gear set, by mean of finite element method. Contact stresses which have very important role in evaluating the service life of gears were obtained and compared with the calculated values by ISO standards. The results showed that acceptable stresses can be obtained by FE model. Furthermore, FEA model with a crack tooth in planet gear were built and analyzed. The main goal was to obtain the stress intensity fac- 
tors and prediction of crack growth path. It was determined that the crack tends to grow through the gear root which is not considering a catastrophic failure, where the crack grows through the gear rim. Similar results were achieved by using XFEM-LEFM based. Furthermore, rim thickness effect was determined by choosing three thicknesses as samples for thick, medium and thin thickness of the rim. It is obvious that by decreasing the rim thickness probability of catastrophic failure due to internally crack growth enhance. This was due to an inadequate amount of rim material which was needed to support the loaded tooth.

\section{References}

[1] Master Thesis, O., 2004, "Stresses and deformations in involute spur gears by finite element method". MS Thesis, University of Saskatchewan.

[2] Chen, $Z$ and Shao, $Y$, "Dynamic simulation of spur gear with tooth root crack propagation along tooth width and crack depth". Engineering Failure Analysis, 18(2011) 2149-2164.

[3] Ristic, D, "Numerical model for the critical stress determination in spur gears, 2009.

[4] Manual of ABAQUS finite element analysis software package.

[5] ISO 6336, 1998.

[6] Lewicki, D, G, "Effect of rim thickness on gear crack propagation path", Army Research Laboratory, 1996. 